\title{
Creatividad, Sueño y Transformación Educativa. La participación de la comunidad en la etapa del sueño de una Comunidad de Aprendizaje
}

\author{
Lorena Adame García. Universidad Valladolid \\ María Jesús Márquez García. Universidad de Málaga
}

Recepción: 17.10.2018| Aceptado: 22.11.2018

Correspondencia a través de ORCID: Lorena Adame García

iD 0000-0003-1177-5314

Citar: Adame Garcia, L. y Marquez Garcia, MJ. (2018). Creatividad, Sueño y Transformación Educativa. La participación de la comunidad en la etapa del sueño de una Comunidad de Aprendizaje. ReiDoCrea, 7, 363-375.

\begin{abstract}
Resumen: Las comunidades de aprendizaje suponen transformar la cultura, el aprendizaje y la organización de la escuela contando con la participación de toda la comunidad. La transformación pasa por un proceso de sensibilización, formación, toma de decisiones para iniciar un proceso de sueño colectivo. Éste es un momento en el que toda la comunidad participa en concretar la escuela que quieren y desean construir entre todos y todas como lugar común. Nuestro objetivo es conocer y comprender el proceso de transformación de una comunidad de aprendizaje profundizando en el proceso del sueño desde las voces de participantes de todos los sectores. La investigación se realiza desde el paradigma cualitativo con enfoque de estudio de casos comunicativo, basándonos en la entrevista como instrumento de recogida de información y diálogo igualitario. Las aportaciones de las entrevistas ponen de manifiesto la importancia de la participación de toda la comunidad en el sueño, que se traduce en un proceso emocional democrático movido por la alegría e ilusión de decidir y participar en el funcionamiento del centro educativo haciéndolo propio. Las aportaciones de todos en la organización y el aprendizaje basadas en las interacciones emocionales va configurando un modo diferente de vivir y sentir la escuela.
\end{abstract}

Palabras clave: Participación comunitaria | Sueño

Creativity, Dream and Educational Transformation. The participation of the community in the dream process of a Learning Community

Abstract: Learning communities will transform culture, learning and school's organization when including the entire community. The transformation includes a sensitization's process, decision-making to start a collective dream process. This is a time when the entire community participates to define the school they want and wish to build all together as a shared place. Our aim is to know and understand the transformation process of a learning community by delving into the dream process from participating voices of all sectors. The research is done by the qualitative paradigm with an approach based on communicative case study, using interviews as an instrument for collecting information and egalitarian dialogue. The interviews provide input that indicates the importance of the participation of the entire community in the dream, which can be translated into an emotional democratic process moved by joy and hope to decide and take part in the operation of the educational centre, making it their own. The contributions based on affective interactions about the organization and learning are shaping a different way of living and feeling school.

Keywords: Community Participation | Dream

\section{Introducción}

La necesidad de que las escuelas sean un espacio inclusivo, seguro y de aprendizaje y convivencia para niños y niñas es una demanda generalizada para muchas familias, profesorado y ciudadanía en general. Las escuelas democráticas así constituidas permitirían garantizar el éxito escolar de todo su alumnado, cambiando el sentido de las relaciones y la vida en los centros educativos, es decir, replantear todos sus espacios y prácticas para una participación real de toda la comunidad (Apple y Beanne, 1997; Feito y López, 2008). 
En este sentido, el proyecto de Comunidades de Aprendizaje ${ }^{1}$ trabaja por un proceso de transformación social y cultural del centro educativo y de su entorno, basado en el aprendizaje dialógico y la participación en todos los espacios y momentos, desde el aula al centro y desde el alumnado, el profesorado y familiares al entorno social. Por lo tanto desaparecen las fronteras entre dentro y fuera de la institución escolar siendo posible una intervención global basada en la participación y el aprendizaje (Valls, 2000). Suponen un cambio radical en el modo de participación de las familias, el profesorado y el alumnado en el centro escolar, y un modo diferente de compartir una visión conjunta de un servicio público en el que toda la comunidad forma parte de la organización, gestión y toma de decisiones (Included, 2011). Esta participación involucra a toda la comunidad tanto en los procesos evaluativos como en la toma de decisiones y en todos los procesos educativos del centro escolar. Se aleja de modelos obsoletos de participación de familias de tipo informativo y consultivo, es decir, de aquellos modelos en el que las decisiones las toman unos pocos para todos los demás y sólo se informa de dichas decisiones o se consultan una vez tomadas. Estos modelos de organización jerárquica no promueven el debate, no tienen en cuenta la diversidad de puntos de vista, ni la posibilidad de propuestas consensuadas y profundamente dialogadas (Bonell, 2015).

Junto a la participación comunitaria el objetivo del proyecto de $\mathrm{CdA}$ es mejorar el aprendizaje de todo el alumnado desde un punto de vista inclusivo. Para ello el espacio del aula se convierte en un lugar donde todas las personas pueden aprender y enseñar al igual que en otros espacios del centro, tanto en horario lectivo como no lectivo. Por lo tanto, este proyecto precisa de una coordinación de todos los agentes educativos, en un entorno concreto, cada uno con su historia, su cultura, su visión del mundo, pero que se aporta y se comparte con los demás con la única finalidad de conseguir los mejores procesos de aprendizaje para todos los niños y niñas, es decir, trabajar por la igualdad desde el respeto de las diferencias.

Varias son las etapas para la transformación de un centro en $\mathrm{CdA}$, de entre ellas destacamos la sensibilización. Es el primer momento, en ella se informa sobre el proyecto al profesorado y a las personas interesadas de la comunidad; la segunda es la toma de decisiones, en esta etapa la mayoría de profesorado del claustro tiene que estar de acuerdo en comenzar el proyecto de transformación del centro, teniendo en cuenta los principios dialógicos y participativos de aprendizaje en el aula y la comunidad. La tercera etapa es la del sueño, en ella nos vamos a detener. La etapa del sueño es el momento de mayor participación en la transformación de la comunidad y el punto inicial de un cambio de modelo organizativo. En esta fase participa toda la comunidad para soñar la escuela que desean y necesitan, familias, alumnado, profesorado y todos aquellos agentes del entorno que van a aportar recursos inclusivos al centro educativo.

La etapa del sueño supone el punto de partida de un nuevo proyecto comunitario del centro. Pero el sueño no solo es una etapa, supone un proceso de información sensibilización, movilización y de acercarse y llegar a todas las personas de la comunidad. Es decir, para que el sueño sea un buen punto de partida, debemos recoger todos los puntos de vista, toda la diversidad del contexto y debe ser un momento de apertura y reflexión con el barrio y sus agentes sociales, con el mayor número de familias y con todo el profesorado y el alumnado; desde infantil hasta el último curso de primaria. Durante la epata del sueño la escuela pasa a ser un espacio de reflexión, diálogo y participación (Jaussi; Flecha \& Torrego, 2012; Jaussi \& Vega 2004; Elboj et alt, 2002).

\footnotetext{
${ }^{1}$ En adelante $\mathrm{CdA}$
} 
La etapa del sueño es el punto de partida del cambio del centro. Es el momento en el que cambia organizativamente, todas y todos son necesarios para invitar al sueño y organizar el proceso de participación colectiva y, como no, organizar "la fiesta de la democracia". Las etapas que siguen al sueño arrancan de este momento y son la selección de prioridades, la planificación y puesta en marcha. En estas tres etapas se baja al día a día, al funcionamiento de un centro y se van seleccionando cuales son las prioridades que salieron en el sueño, planificando el trabajo y organizando el funcionamiento. El nuevo marco organizativo que se propone y se pone en marcha en el proyecto de CdA, es el de las Comisiones Mixtas que suponen, junto a la fase del sueño, el motor democrático de funcionamiento del centro (Márquez, Ibañez y Padua, 2014).

Como hemos dicho nos detenemos ahora en la dimensión y complejidad que el proceso de transformación y la fase del sueño llevan consigo y nos basamos en un estudio de casos que ahora presentaremos, no sin añadir que de las etapas señaladas destacamos el entramado de pensamientos, acciones y emociones de los implicados, la ilusión compartida y el trabajo colectivo para conseguir la máxima participación que configuran los ingredientes básicos para la puesta en marcha de un cambio democrático. En este estudio de casos nos detenemos en analizar, a partir de las entrevistas realizadas a participantes, la etapa del sueño de un centro educativo en concreto y cómo se vivió este proceso para iniciar un proyecto de aprendizaje inclusivo y participativo.

\section{Acerca del contexto del centro educativo y el proceso de sueño}

La comunidad de aprendizaje en la que realizamos el estudio está situada en un barrio humilde que tradicionalmente se ha dedicado a la agricultura y la artesanía en una ciudad de Castilla y León. Desde hace algunos años, se atribuye al bajo precio de la vivienda el motivo principal para la llegada de población de origen inmigrante, en su mayoría marroquí. No solamente ha cambiado la población del barrio sino también la actividad económica pasando a ser, en la actualidad, el sector servicios y el pequeño comercio la principal ocupación de la zona.

El centro educativo imparte el segundo ciclo de educación infantil (3-6 años) y la educación primaria (6-12 años). Tiene doble línea en todos los niveles y cuenta con más de 300 estudiantes y 30 docentes. Cabe destacar que, cómo hemos mencionado, el $20 \%$ del alumnado es de origen inmigrante. Es un centro educativo, caracterizado por la diversidad de su alumnado y su capacidad innovadora. En la década de los 90 ya consiguieron desarrollar un aula de compensatoria dirigida a las minorías étnicas que recién estaban llegando. Más adelante, desarrollaron un programa de acogida para alumnos inmigrantes, pero todas estas medidas resultaban insuficientes. El profesorado verbalizaba que pese al esfuerzo, de docentes y centro, por ofrecer una educación de calidad, el sentimiento que existía era que no se lograban resolver las necesidades del alumnado. Muchos de los vecinos del barrio no querían llevar a sus hijos e hijas a este centro debido al gran número de alumnado de origen inmigrante que había en el centro. Otro problema que tenía el centro era la constante incorporación durante todo el curso escolar de alumnado, en algunos cursos se podían llegar a incorporar en torno a 30 nuevos alumnos/as. Este alumnado, recién llegado, generalmente presentaba desconocimiento de la lengua española y situaciones curriculares diferentes que hacía que el profesorado lo viviera con inquietud.

Durante estos años los docentes no dejaron de buscar y poner en marcha nuevas medidas, invadiéndole un sentir de estar trabajando sin un rumbo claro, como si fueran encadenando "improvisaciones". La llegada de la L.O.E. y sus competencias les hace 
claramente ver que, con las características de su centro, no podrían optar a convertirse en un centro bilingüe tal y como era la tendencia de otros centros en ese momento. Fue a partir de tomar conciencia de esta situación cuando se interesan por conocer el proyecto de Comunidades de Aprendizaje. Como señala la directora, en las entrevistas realizadas, es entonces cuando "ven una luz", un proyecto en el que quieren profundizar y que reúne una visión global de cambio.

La propuesta de transformación en CdA fue del equipo directivo. En un claustro de profesores el equipo directivo comparte esta propuesta al resto del profesorado y comienza la fase de formación. Ellos cuentan que este momento fue vivido con gran ilusión por una gran mayoría del claustro. Poco a poco fueron descubriendo, compartiendo y dialogando sobre medidas de éxito educativo. En líneas generales existe un acuerdo sobre el éxito de esta etapa aunque el equipo directivo señala que fue una etapa de muchas dudas e incertidumbres, que llevaron a generar un rico debate. Para el profesorado esta fase resultó interesante, intensa e intensiva y cargó de ilusión al claustro para las etapas posteriores. Por el contrario para el alumnado y las familias esta fase no fue tan visible, aunque formaron parte de ella personas e instituciones que tenían vinculación con el centro (ONGs, personal religioso, familiares,...). Una vez realizada la sensibilización y formación llega el momento de decidir si tomar o no la decisión de transformarse. Para tomar tan difícil decisión es necesario establecer un intenso debate entre toda la comunidad donde compartir toda la información y formación recibida en la anterior etapa. Es un momento donde emergen muchas dudas. La decisión debe ser conjunta y cada centro tiene libertad de organizarse como consideren más adecuado a su contexto.

El profesorado recuerda dos momentos importantes en el proceso de transformación en Comunidad de Aprendizaje. Por una parte la toma de decisión del profesorado, y por otro la toma de decisión con las familias. Relatan que era una tarde lluviosa de finales del mes de febrero y la respuesta recibida por parte de las familias desbordó las expectativas de los docentes. No quedaban sillas libres pero lo más relevante de todo lo vivido en aquella reunión fue la unanimidad mostrada por todos y todas en la firme voluntad de seguir adelante con este proyecto. La transformación ya era visible en el centro e imparable. La implicación de las familias en esta fase quedó reflejada en la alta participación de las mismas en la votación, aunque no todas las familias eran muy conscientes de en qué consistía una Comunidad de Aprendizaje y albergaban muchas dudas al respecto.

Ahora vendría el momento más esperado, el sueño de todos y todas. Las primeras cuestiones que se platearon era: ¿Qué debemos soñar? Debemos soñar con la escuela que deseamos, aquella en la que no existen limitaciones y ¿Cómo lo hacemos? ¿Cómo diseñamos el proceso de sueño para llegar a todos y todas?. Esto no es sencillo. Supone la participación de toda la comunidad y cada centro debe diseñar su propia estrategia para conseguir la máxima participación.

En líneas generales cada centro que se implica en esta transformación diseña su fase del sueño, respondiendo a las necesidades y características de su entorno. En algunas ocasiones las familias, el profesorado y el alumnado soñaran por separado estableciendo un diálogo comenzando a llevar a cabo los principios del aprendizaje dialógico. Otros centros decidirán establecer un punto de encuentro común en el que quedaran plasmados y recogidos los sueños de toda la comunidad. Luego es necesario dinamizar este proceso de forma diferente pero con un objetivo común soñar cómo nos gustaría que fuera la escuela de nuestra comunidad. Todos los sueños son compartidos con la comunidad y generalmente quedan recogidos en algún espacio común. Esta fase suele finalizar con una gran fiesta que simboliza la nueva 
apertura del centro a la comunidad y con el sueño se comienza a vivir en esta nueva escuela. En esta etapa el reto es superar una práctica mayoritaria en la que el profesorado impone y decide a qué deben o no aspirar su alumnado y familias por otra en donde todos los implicados tienen posibilidad de decidir cuál es la escuela que desean. El sueño abre la posibilidad a participar a cualquier individuo (Freire, 1992; Flecha, 2015; Flecha \& Torrego, 2012; Munté \& Pulido, 2009; Jaussi \& Vega, 2004; Fernández, 2014)

En el estudio que presentamos decidieron que un gran árbol colocado a la entrada del centro sería el eje vertebrador y la forma de dar visibilidad a los sueños de la comunidad. El primer acuerdo que se tomó fue precisamente homenajear, a través del árbol de los sueños, al pedagogo que da nombre al centro. Una vez que el gran árbol fue colocado en la entrada principal llegó el momento de explicar a todos y todas cómo comenzaríamos a soñar. El alumnado fue informado previamente y junto con ayuda de su profesorado fueron llenando las ramas del árbol de sueños. A las familias se les explicó qué era el árbol de los sueños y se les invitó a soñar, todas las personas que de uno u otro modo trabajaban en el centro también tuvieron su oportunidad de soñar. Así mismo, el profesorado y el equipo directivo fueron los encargados de dinamizar todo este proceso invitando a todas las personas posibles. La directora nos cuenta que fueron dos intensos meses donde toda la comunidad entraba y salía a depositar sus sueños. Pero ese ir y venir de personas tuvo su gran momento en la fiesta del sueño, un día con mucha magia, ilusión y donde el cole se llenó de camisetas y globos azules para dar a conocer el árbol de los sueños. Se prepararon multitud de sorpresas para la ocasión, y se movilizaron a jóvenes y mayores del barrio para vivir ese gran momento. Acudieron al evento más de 600 personas y se culminó con una gran macarronada preparada con la colaboración de padres y madres del cole. Esos macarrones eran muy especiales ya que estaban preparados con carne halal para que todas las personas del barrio pudiéramos disfrutar de ellos y algunas personas que aún no habían soñado, pudieron hacerlo en aquella jornada. Aquellos sueños quedaron ordenados en diversas temáticas: Los relacionados con tener una nueva biblioteca y con actividades relacionadas con la lectura; los que hablaban de trabajar de forma cooperativa y sin excluir a ningún alumno del grupo aula; los que recogían la necesidad de una mejora en los sistemas de comunicación centro- familias, utilización de las redes sociales, y difusión, apertura y conocimiento de las actividades del centro; los que hacían referencia a mejorar la estética de los espacios del centro y mejorar sus infraestructuras. También había sueños relacionados con hacer extensivo el aprendizaje a toda la comunidad como más cursos específicos y actividades de implicación de todo el centro.

El centro educativo pone en marcha un proceso de creatividad centrada en los sueños para alcanzar la participación de toda la comunidad. La creatividad en la pedagogía está relacionada con la creatividad en la política; una pedagogía autoritaria y cerrada, al igual que en cualquier otra práctica política o comunitaria, no deja lugar a la libertad necesaria para la creatividad, para aprender y transformarse a uno/a mismo/a y en comunidad (Freire, 2014). En esta línea creatividad y transformación van de la mano en la experiencia de aprendizaje en un contexto de interacción dialógico y participativo (Márquez, Palomero \& Tello 2016).

\section{Objetivos o hipótesis}

El objetivo principal de esta investigación se centra en conocer desde las voces de los participantes el proceso de transformación en Comunidades de Aprendizaje iniciado en la etapa del sueño. Específicamente nos proponemos describir la sucesión de 
acontecimientos producidos en esta etapa identificando las emociones que produce la transformación y acciones que se llevan a cabo en este proceso.

\section{Métodos}

El estudio se enmarca en el paradigma cualitativo, desde un enfoque de estudio de casos comunicativo que nos permite "comprender las complejas relaciones entre todo lo que existe" (Stake 1998, p. 42). Para poder lograr una comprensión completa de la realidad como investigadores/as cualitativos debemos ir más allá de nuestro propio punto de vista o de las teorías existentes (Massot, Dorio, \& Sabariego, 2004, p. 330). Para ello vamos a "buscar una mayor proximidad a la situación y el contacto directo con los participantes para captar su perspectiva personal, alineándose con su propia realidad, y compartiendo sus experiencias y sus actitudes" (Massot et alt., 2004, p. 330). Este enfoque nos permite situar nuestra investigación en un caso concreto con unas condiciones espacio- temporales específicas (Flick, 2004).

El estudio trata un caso concreto y entendido como un sistema acotado (Stake, 1998, 2005) en el que buscamos conocer y comprender un centro educativo y cómo se vivencia el proceso de transformación en CdA. Como afirma Stake (1998) "no es probable que un ejemplo positivo establezca ni modifique una generalización, pero puede incrementar la confianza de los lectores en su generalización" (p. 20). En esta línea Barba (2013) añade que "lo particular siempre tiene algo de lo general, con lo que a medida que diferentes investigaciones concretas van obteniendo resultados similares, la ciencia de la educación avanza consiguiendo acuerdos sobre el campo de estudio" (p. 35).

Por su parte el enfoque comunicativo las personas, mediante la capacidad de lenguaje y acción, usa la capacidad comunicativa, la argumentación y las pretensiones de validez para coordinarse, actuar y cambiar la realidad y las dinámicas sistémicas (Habermas, 1987). Por lo tanto, la realidad se conoce mediante procesos basados en la intersubjetividad y también, como indica Beck (1997), en la reflexión y autorreflexión. Otra premisa de este enfoque es la horizontalidad en las interpretaciones tanto de quienes investigan como de quienes participan en la investigación, ya que como indica Habermas (1987, p.167) los participantes disponen de una competencia de interpretación igual de compleja que la del observador/a. Este enfoque se centra en el diálogo igualitario ${ }^{2}$ entre ciencia y sociedad: la comunidad científica contribuye con los conocimientos del ámbito científico académico y las personas participantes lo hacen desde sus experiencias e interpretaciones (Aubert, Melgar y Valls, 2011).

La perspectiva comunicativa centra el análisis de la información en la superación de las desigualdades sociales a través de una reflexión crítica sobre las dimensiones exclusoras y transformadoras (Flecha \& Soler, 2013) y así contribuir a la construcción de una ciencia social de la posibilidad (Gómez González et alt, 2011). "Las dimensiones exclusoras son aquellas barreras que algunas personas o colectivos encuentran y que les impiden incorporarse a una práctica o beneficio social (Gómez Alonso et alt., 2006, p. 95). En esta investigación las prácticas o beneficios sociales remiten a la organización democrática de la escuela y a la mejora del aprendizaje de la comunidad. Por lo contrario las barreras exclusoras consisten en los elementos que influyen negativamente en el desarrollo de procesos democráticos y participativos para el cambio de la organización.

\footnotetext{
${ }^{2}$ El Dialogo Igualitario es cuando el valor de las aportaciones de los participantes depende de argumentos de validez y no del poder que se ocupa (Aubert, Duque, Fisas \& Valls 2004, Elboj et alt. 2002).
} 


\section{Instrumentos de recogida de Información}

La orientación comunicativa de este trabajo se relaciona con el uso de instrumentos de recogida de datos así como con el análisis y validación de las interpretaciones por parte de los participantes. Los instrumentos empleados para la recogida de información de este estudio ha sido la entrevista apoyada por observaciones comunicativas.

Las entrevistas comunicativas tal y como señalan Gómez González \& Holford (2010, p. 26) "se combina el conocimiento científico y la voz de las personas participantes en los espacios de diálogo igualitario que se generan durante el proceso investigador". En total se llevaron a cabo 13 entrevistas comunicativas. La mayoría fueron individuales (11) y dos de ellas fueron grupales. Entre las entrevistas individuales cinco se realizaron entrevistas en profundidad $y$ seis focales sobre el proceso de transformación.

También utilizamos la observación comunicativa para introducirnos como participantes en la realidad que nos posibilitó obtener una información con mayor profundidad del caso. Lo que caracteriza a la observación comunicativa es su finalidad de compartir y contrastar las interpretaciones sobre lo sucedido en tareas de la vida cotidiana en un plano de igualdad entre la persona observada con la persona investigadora. Ambos a través del diálogo igualitario consensuan e interpretan las informaciones.

\section{Participantes}

El estudio se ha llevado a cabo en un colegio de educación infantil y primaria público de Castilla y León que se ha transformado en CdA. Los participantes del estudio representan diferentes miembros de la Comunidad de Aprendizaje, que al mismo tiempo, participan activamente en alguna de las comisiones mixtas ${ }^{3}$ del centro. Se entrevistan a dos miembros del equipo directivo, tres profesores/as del centro, tres madres, dos alumnas del centro ( $3^{\circ}$ y $6^{\circ}$ de primaria), dos alumnos universitarios que colaboran como voluntarios y un profesor de la universidad que apoya la $\mathrm{CdA}$ desde el asesoramiento y colaboración Universidad-Escuela pública.

\section{Resultados}

Tomando como punto de partida los elementos transformadores que emergen de las entrevistas realizadas. Vamos a presentar las diversas voces con sus aportaciones más significativas en torno al proceso de transformación y sueño.

\begin{tabular}{ll}
\hline Participante & Aportaciones sobre el proceso de transformación y sueño \\
\hline Alumno & (...) Yo me imagino que habia un poco de las dos cosas (miedo e ilusión) tiene mucho mérito porque el ser humano yo \\
Universidad & creo que mucho más aqui (...) se es reticente a los cambios a la gente le cuesta mucho cambiar y haberse mantenido \\
& estables en lo que estaban haciendo y más siendo funcionarios ....es valiente. \\
& (...) Que una escuela pública se mueva de esa forma es súper complicado y más en las zonas interiores \\
& (...) Habia ilusión eso, está claro...porque sin ilusión no existe el cambio pero también habia miedo y habia temor en el \\
& sentido en el que te enfrentas a algo desconocido que no sabes si va a terminar de funcionar...que dependes de la \\
& comunidad educativa, que dependes de que los padres se muevan y entren en las aulas para que haya grupos \\
& interactivos...
\end{tabular}

\footnotetext{
${ }^{3}$ Las comisiones mixtas son espacios de decisión democráticos cuya función primordial es lograr los objetivos previamente prefijados y planificar actuaciones orientadas a las prioridades de cada centro. Por lo tanto, suponen una transformación de los espacios de decisión modificando las estructuras organizativas tradicionales por otras más democráticas (Barrio 2005, Aubert et alt.2008, Elboj et alt. 2002).
} 
Profesorado $\quad$ (...) La formación estuvo muy bien, fue muy interesante, muy intenso, muy intensivo

(...) Fue complicado no todo el mundo estaba de acuerdo en cambiar a comunidades de aprendizaje,

(...) Yo personalmente me ilusioné desde el principio.....pero bueno tuvimos nuestros más y nuestros menos mucho diálogo en el claustro de profesores la gente que estábamos a favor y en contra y mucho intercambio de opiniones lo cual fue muy enriquecedor

(...) Luego al final llegamos al consenso de que sí no todo el mundo pero muy pocas personas dijeron que no, pero como hubo un número mayoritario

(...) Como a mí me ilusionaba [el proyecto de Comunidades de Aprendizaje] en aquel momento empecé a trabajar por él y empezamos a trabajar en el sueño del cole que queríamos

(...) Un momento apasionante, una maravilla lo vivimos con muchísima ilusión

(...) la fiesta del sueño fue preciosa

(...) la apertura del cole a la comunidad fue sorprendente, todos estábamos encantados.

(...) Lo que ocurre es que la fiesta del sueño es muy excitante muy emocionante pero luego ya hay que ponerse a trabajar y cuesta un poco ....había que prepararse, ....y aunque es muy sencillo hay que acostumbrarse.

(...) Yo lo que te estaba diciendo que no teníamos ni idea, que lo hicimos como formación...(nos costó, nos costó)

(...) Buaa a nivel personal que te voy a contar...uno no está en estas cosas porque le obliguen está porque te convence, te llena, se sirve para tu carrera de profesor...

Saber que trabajamos en una línea es muy importante....saber que todos trabajamos en lo mismo ....que te voy a decir satisface tanto te hace sentir tan guay vienes al colegio con ganas...muchísima satisfacción

(...) Todo lo que me aporta a mi es enormemente más de lo que yo aporto a esa comisión

(...) Los consejos escolares eran espacios abiertos para hablar y proponer, los consejos escolares ha habido sitios que han funcionado muy bien pero esto...es diferente en qué sentido es diferente trabajamos un ámbito y todo el mundo habla en el mismo nivel...el alumnado, el conserje, el personal de comedor, las profes, las madres,...

(...) Trabajamos los sentimientos que ahora está muy de moda y en todos los sitios se hace pero nosotros partimos de los sentimientos hace años..

(...) La palabra clave es "Participación" sentirte que formas parte de la comunidad que entonces como formas parte tienes tu hueco y tienes tu hueco no en temas banales sino en decisiones importantes en cosas que van a afectar a todo el mundo

(...) Lo que se ha hablado en la comisión llega y trasciende a los demás entonces tu nivel de participación es parte de la comunidad

(...) ¿Cuándo se siente uno integrado en un sitio? Cuando puede hacer algo por eso, cuando te dejan participar

(...) depende mucho de las personas...para que esto funcione la gente tiene que implicarse y comprometerse si la gente no se implica y no se compromete se queda en agua de borrajas...esto es así

(...) hay gente que no quiere comprometerse y eso así....de lo que se trata de que haya un ambiente de compromiso e implicación...pero nunca es fácil...

\begin{tabular}{|c|c|}
\hline Directora & 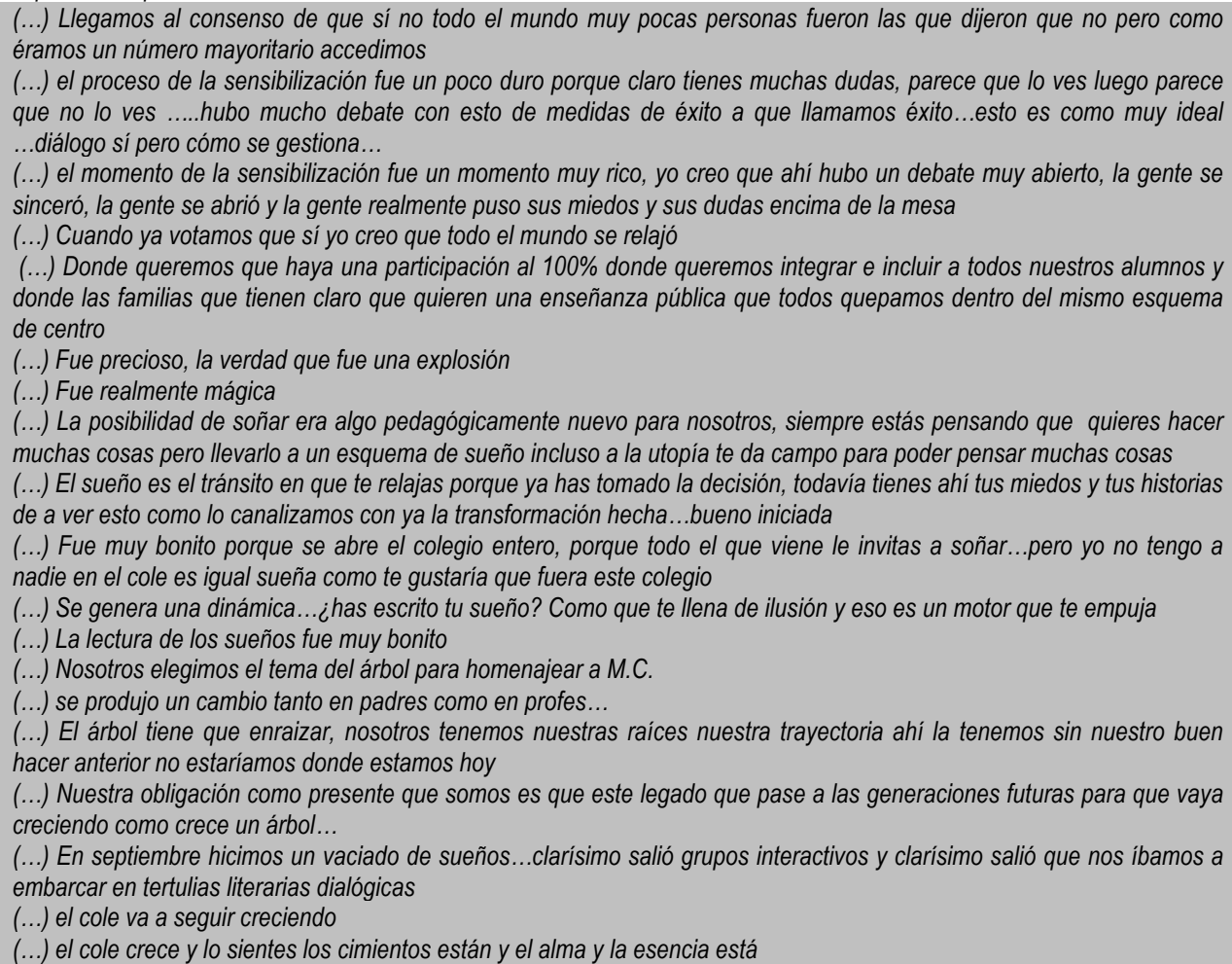 \\
\hline $\begin{array}{l}\text { Profesor } \\
\text { Universidad } \\
\text { Colaborador }\end{array}$ & $\begin{array}{l}\text { (...) La primera que me llamó para hablar del tema de comunidades fue la jefa de estudios...que estaban interesados... } \\
\text { (...) A partir de aceptar llevar a cabo la sensibilización fuimos construyendo la historia de hacer un curso (...) Participó casi } \\
\text { todo el mundo (la formación) hubo personas reticentes pero si que se aceptó mayoritariamente } \\
\text { (...) La experiencia de (otro centro) sirvió de mucho porque cada dia que habia grupos interactivos o tertulias pues } \\
\text { viajaban 3-4 maestros/as a verlo alli... } \\
\text { (...) se aprovechó para abrir (la sensibilización) estuvieron familias, voluntariado, gente de la mezquita, personas de } \\
\text { asociaciones que trabajaban en el centro pues también participó (...) Yo estuve en todas las sesiones...lo bueno que tenía } \\
\text { es que la gente no se callaba cuando tenía alguna duda o es más le parecia mal una cosa y estaba en contra...pues } \\
\text { hablaba y esto yo creo que fue muy importante. } \\
\text { (...) había una parte del profesorado que era muy partidaria y otra que era escéptica (...) yo creo que hay que agradecer al }\end{array}$ \\
\hline
\end{tabular}




\begin{tabular}{|c|c|}
\hline & $\begin{array}{l}\text { profesorado escéptico que hablase ...que si se hubiera quedado en silencio pues no nos hubiéramos podido enterar de } \\
\text { cosas ni hubiéramos podido dar los argumentos necesarios para que se impulsara todo eso } \\
\text { (...) abrir el centro y trabajar con el voluntariado es lo que más reticencias generaba pero se sometió a votación. Se } \\
\text { realizó a mano alzada, y aquello fue un bosque de manos en alza... } \\
\text { (...) La fase del sueño fue espectacular [...]una explosión de camisetas y globos azules } \\
\text { (...) a mi me asombró la participación que tuvo, fue energética y muy trabajada } \\
\text { (...) La fiesta del sueño fue una exhibición de fuerza [...] y estéticamente muy bonita }\end{array}$ \\
\hline Madre & $\begin{array}{l}\text { (...) Yo venía a una escuela tradicional ....ni mucho menos me podía imaginar que iba a pasar la revolución que ya había } \\
\text { oido en otro centro } \\
\text { (...) Para un padre poder participar asi me parece una cosa súper enriquecedora } \\
\text { (...) La verdad que el colegio nos convocó como diciendo vamos a hacer un cambio en el colegio como que era como } \\
\text {....que pasa aqui que va a pasar en el cole ....entonces fue una afluencia masiva de padres. Lo explicaron lo que iba a ser } \\
\text { y todo el mundo votó que sí...muchos votaron que si y salian diciendo y eso de comunidad de aprendizaje que es...pues } \\
\text { que los padres podamos entrar más en el colegio y participar más de la educación de nuestros hijos } \\
\text { (...) Para mi esa fue la parte más bonita (...) Miles de sueños...cuando tienes que soñar miles de cosas } \\
\text { (...) Los niños todos eran ... yo quiero una piscina en el colegio } \\
\text { (...) la biblioteca fue un sueño que ahi lo tenemos... } \\
\text { (...) Participar en las clases de tu hijo, era entrar a organizar las cosas del colegio... } \\
\text { (...) cuando mi hija dentro de unos años recuerde esto ....y diga es que nosotros hemos estado alli...sientes mucha } \\
\text { satisfacción }\end{array}$ \\
\hline Alumna & $\begin{array}{l}\text { (...) yo siento como que se está haciendo el colegio más mayor y cada vez la gente más sabe de él, los padres vienen a } \\
\text { saber más de él...por ejemplo a veces,...e....en el segundo trimestre vinieron los de bachillerato para ver un } \\
\text { poquito...hacian apuntes de la tertulia, o grupos interactivos o de la semana del juego....de todo lo que haciamos y pues } \\
\text { bueno siento como que el colegio se hace mucho más mayor cada vez. } \\
\text { (...) pues te sientes muy bien, muy contenta porque te sientes como que has mejorado el colegio que estas ayudando un } \\
\text { montón que ..que has hecho de todo...que has hecho que el colegio vaya un poquito más mejor y es que te sientes muy } \\
\text { bien porque tus ideas por ejemplo propones una idea o una propuesta pues te sientes muy bien luego si la hacen dices yo } \\
\text { la he propuesto que la están haciendo y que todo. } \\
\text { (...) Pues que ha sido una experiencia muy bonita que me he llevado muchos regalos que me he llevado muchas cosas, } \\
\text { me he llevado muchas sorpresas muchos amigos muchos compañeros muchos profesores me llevo un montón de cosas y } \\
\text { creo que va a ir mucho mejor puede que cada vez va creciendo más y va a ir muchísimo mejor. Que el colegio cada vez } \\
\text { crece más hay cosas mejores. }\end{array}$ \\
\hline
\end{tabular}

\section{Discusión}

La escuela y su marco organizativo está relacionado con el modo de comprender el aprendizaje, entendiendo este como un proceso colectivo que se da en el seno de un grupo o una comunidad. Por lo tanto "la educación es una forma de vivir y participar en una cultura determinada" (Rivas, 2003, p. 113). En este sentido la experiencia cultural compartida comienza siendo el ingrediente imprescindible para el aprendizaje. La organización tiene todas las características de cualquier grupo social tales como comunicación, valores, rituales, formas de comportamiento, etc. Por otra parte, los procesos psicológicos de las personas, según los aportes de la psicología cultural, son el resultado de las interacciones y relaciones, subyaciendo la cultura que compartimos. De igual modo las emociones son parte del modo subjetivo en el que nos narramos en relación a la cultura y la política de los contextos que vivimos (Rivas, 2003, Rivas \& Herrera, 2009).

El proceso de transformación del centro en CdA pasa por un cambio cultural y de relaciones expresada y narrada por los participantes de esta investigación como un cambio radical en el modo de vivir la escuela de modo colectivo. Un modo diferente que expresan en forma de emociones tales como la alegría por la ilusión compartida y por el compromiso de todas las partes para que el proceso de cambio salga adelante. Los cambios hacia una mayor democracia en las organizaciones llevan consigo un proceso emocional y expresivo personal y colectivo en el que se evidencia como se van superando y afrontando las reticencias y las incertidumbres, cómo se refuerzan los argumentos para convencer al otro/a, y de qué diferentes modos se comparten los miedos a las nuevas situaciones junto con las esperanzas.

Las emociones son parte de la deliberación de los procesos de cambio, tal y como señala Nussbaum (2008) "El hecho de que los seres humanos deliberen éticamente sobre cómo vivir supone que las emociones son una parte fundamental de la deliberación moral" (p. 201). En la línea de lo que esta autora plantea, las emociones 
deben de conformar la base de una democracia plural, en relación al bienestar siempre respaldado por la mayoría de las personas. Para Nussbaum es necesario fomentar las emociones positivas, o emociones democráticas disuadiendo aquellas que la impiden como el narcisismo, la intolerancia, la vergüenza, la envidia.... Por el contrario las emociones democráticas profundizan en aquellas que hacen una sociedad y una organización más crítica y justa (Panea, 2017). Las emociones y expresiones que nos narran los y las participantes no solo las comunican con palabras, al hablar del proceso de transformación y adentrarnos en la etapa del sueño la expresión emerge de todo el cuerpo, trasladando en cada entrevista la ilusión vivida con movimientos acompañados de palabras "fue una explosión de camisetas y globos azules" (Profesor universidad).

Los cambios también llevan consigo procesos como profundizar en las argumentaciones, convencer a las personas más escépticas y responder con argumentos fundamentados a rechazos basados en creencias pedagógicas obsoletas o miedos personales. Las narrativas de los participantes en el estudio destacan la importancia positiva de estas reticencias para visibilizar todas las opiniones, así como el esfuerzo que supone buscar nuevos argumento y formas de trasladar el proyecto a quienes aún no lo tienen claro. "El momento de la sensibilización fue un momento muy rico, yo creo que ahí hubo un debate muy abierto, la gente se sinceró, la gente se abrió y la gente realmente puso sus miedos y sus dudas encima de la mesa" (directora) (...) Yo me imagino que había un poco de las dos cosas (miedo e ilusión) tiene mucho mérito porque el ser humano yo creo que mucho más aquí (...) se es reticente a los cambios a la gente le cuesta mucho cambiar y haberse mantenido estables en lo que estaban haciendo y más siendo funcionarios ....es valiente (Estudiante voluntario).

Se expresa la satisfacción personal y profesional entre el profesorado de la universidad y el centro educativo en relación a su vida e identidad docente. Entre las expresiones que se utilizan está la de "lo que aporto es menos de lo que me aportan a mi", del mismo modo este sentimiento de satisfacción se encuentra en la madre cuando nos dice "cuando mi hija dentro de unos años recuerde esto...y diga es que nosotros hemos estado allí...sientes mucha satisfacción". Junto a la ilusión se destaca el entusiasmo por poder estar y aportar, ser escuchado/a y poder proponer. "Buaa!!! $A$ nivel personal que te voy a contar...uno no está en estas cosas porque le obliguen está porque te convence, te llena, se sirve para tu carrera de profesor... Saber que trabajamos en una línea es muy importante....saber que todos trabajamos en lo mismo....que te voy a decir satisface tanto te hace sentir tan guay vienes al colegio con ganas...muchísima satisfacción" (Profesora).

A partir de compartir un proyecto común se hacen alusiones personalizando el centro educativo, del que ya se habla de un modo humanizado, haciendo constantemente referencia a lo que está creciendo el centro, lo bien que se le ve ahora. Del mismo modo se verbaliza el agradecimiento por parte de las familias y el alumnado por poder participar, aportar y ayudar a que el centro crezca "estás ayudando un montón" (Alumna). Desde el inicio se asume que se está viviendo un proceso de aprendizaje conjunto, participar en la sensibilización, toma de decisiones y el sueño nos hace conocer más el funcionamiento del centro, sus necesidades educativas y su complejidad social, aprendiendo todos y todas de todos y todas. En este sentido las interacciones y las relaciones se intensifican construyendo otra cultura escolar. Un proceso en el que las emociones ya se ponen en juego y se comparten.

Por su parte Freire (1992) no entiende la capacidad humana sin esperanza y sueño. La esperanza por sí sola no transforma la realidad, pero el sueño es necesario aunque 
no suficiente, por su parte sin ellos es imposible avanzar en los cambios educativos. "No hay cambio sin sueño, como no hay sueño sin esperanza" (p.116). Tener esperanza es fundamental para cambiar el mundo que nos rodea, el más cercano a nuestro cotidiano. Como expresa la directora "La posibilidad de soñar era algo pedagógicamente nuevo para nosotros, siempre estás pensando que quieres hacer muchas cosas pero llevarlo a un esquema de sueño incluso a la utopía te da campo para poder pensar muchas cosas".

El sueño de la CdA nos presenta diferentes modos de cambiar la cotidianidad del centro y el modo de sentirnos en él como docentes, familias y alumnado. Tal y como hemos señalado los sueños señalan diferentes aspectos como, la estética del aula, el patio y el centro en general, la convivencia, el aprendizaje, la biblioteca etc. Un modo de compartir un sentimiento por un lugar en el que desde el momento en el que puedes soñarlo lo estas habitando. Como señala Freire (1992) "Soñar no es sólo un acto político necesario, sino también una connotación de la forma histórico-social de estar siendo mujeres y hombres. Forma parte de la naturaleza humana que, dentro de la historia, se encuentra en permanente proceso de devenir" (p.111).

\section{Conclusiones}

Visibilizar en este artículo el análisis del proceso de transformación de una Comunidad de Aprendizaje nos ha permitido adentrarnos en las voces de las y los participantes poniendo en diálogo todos los modos de vivirlo y sentirlo. El modo de relatar un proceso colectivo desde la narrativa personal deja una huella emocional expresada que da forma a cómo construimos emociones democráticas que promueven el ejercicio de la ciudadanía una vez vivido (Modzelewski, 2014).

Entre los relatos sobre la experiencia de transformación recogidos podemos decir que la ilusión es el principal modo de expresar la emoción de alegría acompañada de incertidumbre y miedo a lo desconocido que emerge en todo el proceso de transformación de esta Comunidad de Aprendizaje. Por parte del profesorado la ilusión por construir una escuela comunitaria inclusiva se relata desde las primeras etapas de sensibilización siendo más visible en el momento del sueño. Tal y como expresan otros participantes esta ilusión se va contagiando y convirtiendo el centro en un hervidero de debates, encuentros y confidencias en las que se van reflejando las incertidumbres hacia lo desconocido junto con las esperanzas en los cambios.

Las interacciones permiten a la comunidad involucrarse de manera activa en ir preparando espacios para tomar parte en el cambio. ¿Desde cuándo la comunidad puede asistir a una formación junto a los profesores en un centro? ¿Cuándo han soñado su propio centro, el de sus hijos e hijas y el de su profesión? ¿Cuándo se ha organizado entre todos y todas para llevar conseguir hacer realidad la escuela que se sueña? La asistencia de familiares en la formación junto al profesorado nos deja intuir que se trata de un proceso colectivo donde existe interés por lo comunitario. Las puertas del centro permanecerán abiertas a partir de estos primeros momentos. Las reuniones y debates se suceden entre todos los miembros de la comunidad permitiendo otras formas de participar. El lenguaje cambia radicalmente hacia un modo inclusivo. Ahora se habla en términos de posibilidades para todos y todas, de sueños y de utopía, hasta el punto que una convocatoria ordinaria a las familias supone una respuesta masiva de las mismas.

Los y las participantes nos narran cómo se les presentan nuevas oportunidades de participación respondiendo a sus intereses y preocupaciones. El camino del cambio esconde mucho trabajo y compromiso y la mágica fiesta del sueño supone un 


\section{despliegue de nervios y emociones que deben ser canalizadas en las posteriores etapas en la construcción cotidiana de una nueva cultura escolar democrática.}

\section{Referencias}

Apple, M. W., \& Beane, J. A. (1997). Escuelas democráticas. Madrid: Morata [V.O. Democratic schools. Virginia: Association for Supervision and Curriculum Development. 1995].

Aubert, A., Duque, E., Fisas, M., \& Valls, R. (2004). Dialogar y transformar. Pedagogía crítica del siglo XXI. Barcelona: Graó.

Aubert, A., Flecha, A., García, C., Flecha, R., y Racionero, S. (2008). Aprendizaje Dialógico en la Sociedad de La Información. Barcelona: Editorial Hipatia.

Aubert, A., Melgar, P., y Valls, R. (2011). Communicative Daily Life Stories and Focus Groups: Proposals for Overcoming Gender Violence Among Teenagers. Qualitative Inquiry, 17(3), 295-303.

Barba, J. J. (2013). La investigación cualitativa en educación en los comienzos del siglo XIX. En M. Díaz \& A. Giráldez (Eds.), Investigación cualitativa en educación musical (pp. 23-38). Barcelona: Graó.

Barrio, J.L., (2005). La transformación educativa y social en las comunidades de aprendizaje. Teoría de la Educación, 17, 129-156.

Beck, U. (1997). La reinvención de la política: hacia una teoría de la modernización reflexiva. En Beck, U., Giddens, A. \& Lash, S., Modernización reflexiva. Política, transición y estética en el orden social moderno (pp. 13-73). Madrid: Alianza Editorial.

Bonell, L. (2016). La promoción de la participación educativa de las familias en la escuela desde una perspectiva transformadora: un estudio de casos. Tesis doctoral. Universidad de Valladolid

Elboj, C., Puigdellívol, I., Soler, M. \& Valls, R. (2002). Comunidades de Aprendizaje. Transformar la educación. Barcelona: Graó.

Feito \& López (2008). Construyendo escuelas democráticas. Barcelona: Hipatia

Fernández, E. (2014) Comunidades de Aprendizaje: proyecto cultural de la sociedad de la información. Avances en supervisión educativa, 22 ,

Flecha, R. (2015). Comunidades de aprendizaje: sueños posibles para todas las niñas y niños. Aula de innovación educativa, $241,12-16$

Flecha, R., \& Soler, M. (2013). Turning difficulties into possibilities: Engaging Roma families and students in school through dialogic learning. Cambridge Journal of Education, 43(4), $451-465$.

Flecha, R. \& Torrego, L. (2012). Aprendizaje dialógico y transformaciones sociales: más allá de los límites. Lenguaje y textos, 36, 15-24
Flick, U. (2004). Introducción a la investigación cualitativa. Madrid: Morata.

Freire, P. (1992). Pedagogía de la esperanza. Un reencuentro con la pedagogía del oprimido. México DF: Siglo XXI.

Gómez Alonso, J., Latorre, A., Sánchez Aroca, M., \& Flecha, R. (2006). Metodologia Comunicativa Critica. Barcelona: El Roure.

Gómez González, A., \& Holford, J. (2010). Contribuciones al éxito educativo desde la metodología comunicativa. Revista Educación Y Pedagogía, 22(56), 21-30.

Gómez González, A., Puigvert, L., \& Flecha, R. (2011). Critical communicative methodology: Informing real social transformation through research. Qualitative Inquiry, 17(3), $235-245$.

Habermas, J. (1987). Teoría de la acción comunicativa I. Racionalidad de la acción y racionalización social. Madrid: Taurus.

INCLUD-ED Consortium. (2011). Actuaciones de éxito en las escuelas europeas. Madrid: Ministerio de Educación.

Jaussi M.L. (). Comunidades de aprendizaje en la CAPV. Una respuesta educativa en la sociedad de la información para todos y todas. [Archivado por WebCite ${ }^{\circledR}$ en http://www.webcitation.org/730unf8uS]

Jaussi, M.L \& Vega, C. (2004). Construyendo Un Sueño: Comunidades De Aprendizaje En El País Vasco. Networks: An Online Journal for Teacher Research, 7(1)

Márquez, M.J. Palomero, A \& Tello, J. (2016) Creatividad compartida. Narrativa de una experiencia de formación y transformación social. EN Cortés, P. y Márquez, M.J. (eds) Creatividad, comunicación y Educación. Más allá de las fronteras del saber establecido. Málaga. Universidad de Málaga.

Márquez, M.J, Ibañez, L. \& Padua, D. (2014). Comunidades de aprendizaje y éxito educativo. En Moreno A. y Arancibia M. Editores. Educación y Transformación Social: construyendo una ciudadanía crítica. Valparaíso: Ediciones Universitarias de Valparaiso.

Massot, I., Dorio, I., \& Sabariego, M. (2004). Estrategias de recogida y análisis de la información. In R. Bisquerra (Ed.), Metodologia de la Investigación Educativa. Madrid: La Muralla.

Munté, A., \& Pulido, M. Á. (2009). El papel del trabajo social en el sueño de barrio. Revista de La Asociación de Sociología de La Educación, 2(3), 56-65.

Modzelewski, H. (2014) Autorreflexión y educación de las emociones para la Democracia. Entrevista a Martha Nussbaum. Areté. Revista de Filosofía. Vol. XXVI, № 2, pp. 315-333 
Nussbaum, M. C. (2008). Paisajes del pensamiento. La inteligencia de las emociones. Barcelona: Paidós Ibérica.

Panea, J.M (2017) El papel de las emociones en la esfera pública: la propuesta de M. C. Nussbaum. En Recerca, revista de pensament i anàlisi, N. 22. (pp. 111-131).

Rivas, J.I. (2003) La perspectiva cultural de la organización escolar: marco institucional y comportamiento individual. En Educar, 31 (pp. 109-119)

Rivas, J. I \& Herrera, D. (2009) Voz y educación. La narrativa como enfoque de interpretación de la realidad. Barcelona. Octaedro.
Stake, R. E. (1998). Investigación con estudio de casos. Madrid: Morata.

Stake, R. E. (2005). Qualitative Case Studies. In N. K. Denzin \& Y. S. Lincoln (Eds.), The SAGE handbook of qualitative research (3rd ed.). Thousand Oaks: SAGE Publications.

Valls, R. (2000). Comunidades de Aprendizaje: Una práctica Educativa de Aprendizaje Dialógico para la Sociedad de la Información. Tesis doctoral. Universidad de Barcelona 